\title{
Embedding Imputation with Grounded Language Information
}

\author{
Ziyi Yang ${ }^{1}$, Chenguang Zhu ${ }^{2}$, Vin Sachidananda ${ }^{3}$, and Eric Darve ${ }^{1,4}$ \\ ${ }^{1}$ Department of Mechanical Engineering, Stanford University \\ ${ }^{2}$ Microsoft Speech and Dialogue Research Group \\ ${ }^{3}$ Department of Electrical Engineering, Stanford University \\ ${ }^{4}$ Institute for Computational and Mathematical Engineering, Stanford University \\ \{ziyi.yang, vsachi, darve\}@stanford.edu, chezhu@microsoft.com
}

\begin{abstract}
Due to the ubiquitous use of embeddings as input representations for a wide range of natural language tasks, imputation of embeddings for rare and unseen words is a critical problem in language processing. Embedding imputation involves learning representations for rare or unseen words during the training of an embedding model, often in a post-hoc manner. In this paper, we propose an approach for embedding imputation which uses grounded information in the form of a knowledge graph. This is in contrast to existing approaches which typically make use of vector space properties or subword information. We propose an online method to construct a graph from grounded information and design an algorithm to map from the resulting graphical structure to the space of the pre-trained embeddings. Finally, we evaluate our approach on a range of rare and unseen word tasks across various domains and show that our model can learn better representations. For example, on the Card-660 task our method improves Pearson's and Spearman's correlation coefficients upon the stateof-the-art by $11 \%$ and $17.8 \%$ respectively using GloVe embeddings.
\end{abstract}

\section{Introduction}

Word embeddings (Mikolov et al., 2013; Pennington et al., 2014) are used pervasively in deep learning for natural language processing. However, due to fixed vocabulary constraints in existing approaches to training word embeddings, it is difficult to learn representations for words which are rare or unseen during training. This is commonly referred to as the out-of-vocabulary (OOV) word problem. In the original embedding implementations, a special OOV token is typically reserved for such words. However, this rudimentary approach often detriments the performance of downstream tasks which contain numerous rare or un- seen words. Recent works have proposed subword approaches (Zhao et al., 2018; Sennrich et al., 2015), which construct embeddings through the composition of characters or sentence pieces for OOV words. Vector space properties are also utilized to learn embeddings with small amounts of data (Bahdanau et al., 2017; Herbelot and Baroni, 2017). In this paper, we propose a novel approach, knowledge-graph-to-vector (KG2Vec), for the OOV word problem. KG2Vec makes use of the grounded language information in the form of a knowledge graph. Grounded information has been extensively used in various NLP tasks to represent real-world knowledge (Niles and Pease, 2003; Gruber, 1993; Guarino, 1998; de Bruijn et al., 2006; Paulheim, 2017) . In particular, early question answering systems used expert-crafted ontologies in order to endow these systems with common knowledge (Harabagiu et al., 2005; Xu et al., 2016). Additionally, lexical-semantic ontologies, such as WordNet, have been used to provide semantic relations between words in a wide variety of language processing and inference tasks (Morris and Hirst, 1991; Ovchinnikova et al., 2010).

Grounded language information has been observed to augment model performance on a wide variety of natural language processing and understanding tasks (He et al., 2017; Choi et al., 2018). In these settings, a model is able to provide better generalization by using relational information from a knowledge graph or knowledge base in addition to the standard set of training examples. Additionally, outputs from models with grounded approaches have been observed to be more factually consistent and logically sound (Bordes et al., 2014) compared with outputs from models without grounding information.

By foregoing the usage of vector space or subword information, KG2 Vec is able to capture se- 
mantic meanings of words directly from the graphical structure in grounded knowledge using recent advances in network representation learning. Furthermore, KG2Vec leverages the most updated information from comprehensive knowledge bases (Wikipedia \& Wiktionary). Therefore, KG2Vec can be applied to training embeddings of newly emerging OOV words.

In summary, our contributions are three-fold:

1. An approach to constructing graphical representations of entities in a knowledge base in an unsupervised manner.

2. Methods for mapping entities from a graphical representation to the space in which a pretrained embedding lies.

3. Experimentation on rare and unseen word datasets and a new state-of-art performance on Card-660 dataset.

\section{Related Work}

\subsection{Graph Neural Networks}

Graph neural networks (GNN) are an emerging deep learning approach for representation learning of graphical data (Xu et al., 2018; Kipf and Welling, 2016). GNNs can learn a representation vector $h_{v}$ for each node in the network by leveraging the graphical structure and node features $f_{v}$. Node embeddings are generated by recursively aggregating each node's neighborhood information and features. At the $t$-th iteration, the information aggregation is defined as:

$$
h_{v}^{t}=M^{t}\left(h_{v}^{t-1},\left\{h_{u}^{t-1}\right\}_{u \in N(v)}\right)
$$

where $h_{v}^{t}$ is the representation for $v$ at the $t$-th iteration, $M^{t}$ is an iteration-specific message aggregation function parametrized by a neural network and $N(v)$ is the set of neighbors of node $v$. One simple form of $M^{t}$ is mean neighborhood aggregation:

$$
h_{v}^{t}=\operatorname{ReLU}\left(\sum_{u \in N(v)} \frac{W^{t} h_{u}^{t-1}}{|N(v)|}+B^{t} h_{v}^{t-1}\right)
$$

where $W^{t}$ and $B^{t}$ are trainable matrices. Typically, $h_{v}^{0}$ is initialized as $f_{v}$. The final node representation is usually a function of $h_{v}^{T}$ from the last iteration $T$, such as an identity function or a transformation function (Ying et al., 2018).

\subsection{The OOV word problem}

The out-of-vocabulary (OOV) word problem has been present in word embedding models since their inception (Mikolov et al., 2013; Pennington et al., 2014). Due to space and training data constraints, words which are either infrequent or do not appear in the training corpus can lack representations at the time of inference.

Numerous methods have been proposed to tackle the OOV word problem with a small amount of training data. Deep learning based approaches (Bahdanau et al., 2017) and vector-space based methods (Herbelot and Baroni, 2017) can improve the rare word representations on various semantic similarity tasks. One downside to these approaches is that they require small amounts of training data for words whose embeddings are being imputed and, as a result, can have difficulties representing words for which training samples do not exist.

Sub-word level representations have been studied in the context of the OOV word problem. Pinter et al. (2017) uses the RNN's hidden state of the last sub-word in a word to produce representations. Zhao et al. (2018) proposes using characterlevel decomposition to produce embeddings for OOV words.

\section{Model}

We propose the knowledge-graph-to-vector (KG2Vec) model for building OOV word representations from knowledge base information. KG2Vec starts with building a knowledge graph $\mathcal{K}$ with nodes consisting of pre-trained words and OOV words. It then utilizes a graph convolutional network (GNN) to map graph nodes to lowdimensional embeddings. The GNN is trained to minimize the Euclidean distance between the node embeddings to pre-trained word embeddings in the dictionary such as GloVe (Pennington et al., 2014) and ConceptNet Numberbatch (Speer et al., 2017). Finally, the GNN is used to generate embeddings for OOV words.

\subsection{Build the Knowledge Graph}

In a knowledge graph $\mathcal{K}$, each node $v$ represents a word $w_{v}$. The nodes (words) in the graph are chosen as follows. We count the frequency of occurrences for English words from the Wikipedia English dataset (with 3B tokens). The 2000 words with the highest frequencies of occurrence are skipped to diminish the effect of stop words. Among the words left, we choose the $\left|V^{\prime}\right|$ words with the highest frequencies of occurrence. All 
OOV words for which we would like to impute embeddings are also added to the graph as nodes.

For each node, we obtain its grounded information from two sources: (I) the words' summary, defined as the first paragraph of the Wikipedia page when this word is searched; (II) the word's definition in Wiktionary. We choose Wikipedia and Wiktionary over other knowledge bases because they are comprehensive, well-maintained and up-to-date. Here is an example of the grounded information for the word Brexit.

- Wikipedia page summary: Brexit, a portmanteau of "British" and "exit", is the impending withdrawal of the United Kingdom (UK) from the European Union (EU). It follows the referendum of 23 June 2016 when 51.9 per cent of voters chose to leave the EU...

- Wiktionary definition: Brexit (Britain, politics) The withdrawal of the United Kingdom from the European Union.

All the words in the Wikipedia summary and the Wiktionary definition form the grounded language information of this word $w_{v}$, defined as $D_{v}$. Specifically, $D_{v}$ is the concatenation of $w_{v}$ 's Wikipedia summary and the Wiktionary definition. An undirected edge $e_{v u}$ exists between node $v$ and $u$ if the Jaccard coefficient $\frac{\left|D_{v} \cap D_{u}\right|}{D_{v} \cup D_{u} \mid}>\eta$, where $\eta$ is a pre-defined threshold and chosen to be 0.5 empirically in the experiments. The edge $e_{v u}$ is then assigned with a weight $s_{v u}=\frac{\left|D_{v} \cap D_{u}\right|}{\left|D_{v} \cup D_{u}\right|}$. We also compute a feature vector $f_{u}$ as the mean of pre-trained embeddings of words in $D_{v}$. Finally, the obtained knowledge graph $\mathcal{K}=(V, E)$ has a feature vector $f_{v}$ for each node $v \in V$.

\subsection{Graph Neural Network}

The nodes in the graph are mapped to lowdimensional embeddings via graph convolutional neural network (GCN) (Kipf and Welling, 2016). It follows that, at the $t$-th neighborhood aggregation, the node embedding $h_{v}^{t}$ for node $v$ is modelled as:

$$
h_{v}^{t}=\operatorname{ReLU}\left(W^{t} \sum_{u \in S(v)} \frac{s_{v u} h_{u}^{t-1}}{C}+b^{t}\right)
$$

where $S(v)=N(v) \cup\{v\}$, and the normalization constant $C=1+\sum_{u \in N(v)} s_{v u} . W^{t}$ and $b^{t}$ are trainable parameters. The node embeddings are initialized as the feature vector $f_{v}$, i.e. $h_{v}^{0}=f_{v}$. At the final iteration $T$, the generated node embeddings $\left\{h_{v}^{T}\right\}$ are computed without the ReLU func- tion. The loss function of the GNN model is the mean square error between the pre-trained word vectors and generated embedding $h_{v}^{T}$ for all words in the graph which are part of the model's vocabulary (e.g. GloVe). During inference, OOV words are assigned embeddings computed by the GNN.

\section{Experiments}

To evaluate our method's ability to impute embeddings, we conduct experiments on the following rare and unseen word similarity tasks.

\subsection{Card-660: Cambridge Rare Word Dataset}

Card-660 (Pilehvar et al., 2018) is a word-word similarity task with 660 example pairs involving uncommon words and provides a benchmark for rare word representation models. Card-660 has a inter-annotator agreement (IAA) measure of 0.90 , which is significantly higher than previous datasets for rare word representation. Additionally, Card-660 contains examples from a disparate set of domains such as technology, popular culture and medicine.

\subsection{Stanford Rare Word (RW) Similarity}

The Stanford Rare Word (RW) Similarity Benchmark (Luong et al., 2013) is a word-word semantic similarity task including 2034 word pairs and tests the ability of representation learning methods to capture the semantics of infrequent words. Due to the probabilistic underpinnings of word embeddings, where distances between two words' representations are approximately proportional to their co-occurrence probability in a corpus, the authors found that rare words often have more noisy representations due to having fewer training samples. Although RW has a relatively low IAA measure of 0.41 , the benchmark has been well-studied in previous literature.

\subsection{Results}

Experiment results, measured by Pearson's and Spearman's correlation, on the Card-660 and Stanford rare words datasets are shown in table 1 . The Wikipedia pages and Wiktionary definitions used in the following experiments are snapshots from Feb 16th, 2019. We compare KG2Vec to other embedding imputation models, including Mimick (Pinter et al., 2017), Definition centroid (Herbelot and Baroni, 2017), Definition LSTM (Bah- 


\begin{tabular}{|c|c|c|c|c|c|c|c|c|}
\hline \multirow{2}{*}{ Model } & \multicolumn{2}{|c|}{ Missed words } & \multicolumn{2}{|c|}{ Missed pairs } & \multicolumn{2}{|c|}{ Pearson $r$} & \multicolumn{2}{|c|}{ Spearman $\rho$} \\
\hline & RW & CARD & RW & CARD & RW & CARD & RW & CARD \\
\hline ConceptNet Numberbatch & $5 \%$ & $37 \%$ & $10 \%$ & $53 \%$ & 53.0 & 36.0 & 53.7 & 24.7 \\
\hline + Mimick & $0 \%$ & $0 \%$ & $0 \%$ & $0 \%$ & 56.0 & 34.2 & 57.6 & 35.6 \\
\hline + Definition centroid & $0 \%$ & $29 \%$ & $0 \%$ & $43 \%$ & 59.1 & 42.9 & 60.3 & 33.8 \\
\hline + Definition LSTM & $0 \%$ & $25 \%$ & $0 \%$ & $39 \%$ & 58.6 & 41.8 & 59.4 & 31.7 \\
\hline+ SemLand & $0 \%$ & $29 \%$ & $0 \%$ & $43 \%$ & $\underline{60.5}$ & 43.4 & 61.7 & 34.3 \\
\hline$+\mathrm{BoS}$ & $0 \%$ & $0 \%$ & $0 \%$ & $0 \%$ & $\overline{60.0}$ & 49.2 & $\overline{61.7}$ & 47.6 \\
\hline+ Node fea & $0.02 \%$ & $7 \%$ & $0.04 \%$ & $12 \%$ & 58.4 & 54.0 & 59.7 & 51.4 \\
\hline$+\mathrm{KG} 2 \mathrm{Vec}$ & $0.02 \%$ & $7 \%$ & $0.04 \%$ & $12 \%$ & 58.6 & $\underline{56.9}$ & 60.1 & $\underline{54.3}$ \\
\hline GloVe Common Crawl & $1 \%$ & $29 \%$ & $2 \%$ & $44 \%$ & 44.0 & 33.0 & 45.1 & 27.3 \\
\hline + Mimick & $0 \%$ & $0 \%$ & $0 \%$ & $0 \%$ & 44.7 & 23.9 & 45.6 & 29.5 \\
\hline+ Definition centroid & $0 \%$ & $21 \%$ & $0 \%$ & $35 \%$ & 43.5 & 35.2 & 45.1 & 31.7 \\
\hline + Definition LSTM & $0 \%$ & $20 \%$ & $0 \%$ & $33 \%$ & 24.0 & 23.0 & 22.9 & 19.6 \\
\hline+ SemLand & $0 \%$ & $21 \%$ & $0 \%$ & $35 \%$ & 44.3 & 39.5 & 45.8 & 33.8 \\
\hline$+\mathrm{BoS}$ & $0 \%$ & $0 \%$ & $0 \%$ & $0 \%$ & 44.9 & 31.5 & 46.0 & 35.3 \\
\hline+ Node featu & $0.05 \%$ & $0.4 \%$ & $0.01 \%$ & $0.7 \%$ & 43.8 & 36.0 & 45.0 & 37.4 \\
\hline$+\mathrm{KG} 2 \mathrm{Vec}$ & $0.05 \%$ & $0.4 \%$ & $0.01 \%$ & $0.7 \%$ & 44.6 & 50.5 & 45.8 & 51.6 \\
\hline
\end{tabular}

Table 1: Performance of OOV models on Stanford Rare Word Similarity and Card-660 datasets. Two word dictionaries are used: ConceptNet and GloVe. The overall best are underlined for each column, and the best results for each type of word dictionary are in bold. We run the BoS experiments with the default hyper-parameters from Zhao et al. (2018). Performances of other baseline models are collected from Pilehvar et al. (2018).

danau et al., 2017), SemLand (Pilehvar and Collier, 2017) and BoS (Zhao et al., 2018). During evaluation, zero vectors are assigned to missing words and word-word similarity is computed as the inner product of the corresponding embeddings. In KG2Vec, the number of iterations $T=$ 3 for GCN, and the number of nodes with pretrained word vectors $\left|V^{\prime}\right|=9000$. We test on two types of pre-trained word vectors GloVe (Common crawl, cased 300d) and ConceptNet Numberbatch (300d). KG2Vec shows competitive performance in all test cases. On Card-660 dataset KG2Vec achieves state-of-the-art results by a significant margin. When using ConceptNet embeddings, KG2Vec results in improvements of $7.7 \%$ and $6.7 \%$ on Pearson's and Spearman's correlation coefficients, respectively, when compared to prior state-of-the-art performance (BoS). When using GloVe embeddings, KG2Vec improves upon SemLand by $11 \%$ and $17.8 \%$ on Pearson's and Spearman's correlation coefficients. Considering the fact that Card-660 contains a significant amount of recent OOV words (e.g. "Brexit"), this improvement indicates that KG2Vec's can leverage upto-date information from knowledge bases. Additionally, this shows that GNNs can effectively cover OOV words and precisely model their se- mantic meanings. On Stanford Rare Word dataset, $\mathrm{KG} 2 \mathrm{Vec}$ is comparable with other state-of-the-art models, suggesting its robustness across various test schemes. Note that the graph used in KG2Vec has a much smaller size compared with knowledge graphs used in SemLand, the WordNet, which has 155,327 words.

To fairly evaluate KG2Vec, we include a baseline model that assigns the node feature $f_{v}$ as the final word representations for word $w_{v}$ if $w_{v}$ is not in the pre-trained dictionary. The results are denoted as "Node features" in table 1. In all test cases, KG2Vec improves by a large margin upon this baseline. For example, using GloVe on the Card-660 dataset, KG2Vec's achieves a performance increase of $14.5 \%$ and $14.2 \%$ respectively for Pearson's and Spearman's coefficients over Node features. This observation suggests that the information aggregation by GNN is critical for embedding imputation and semantic inference. It also indicates that learning from the knowledge graph and its language information is an effective way to parse the semantic meaning of a rare word.

\section{Discussion}

Application on Entity Relations Knowledge

Base. Many public knowledge bases consist of 
relational data in a tuple format: (entity1, entity2, relation), where entities can be considered as the "nodes" in the graph and relations define the edges. Note that there are different kinds of relations and therefore edges in the graph have different types or labels. To impute the embeddings for entities in such scenario, one can conveniently adapt KG2Vec following Schlichtkrull et al. (2018) by learning different transformations for different types of edges.

\section{Adaption to New Vocabularies and Informa-}

tion. Considering the fast growth of vocabularies in the current era, the ability to perform online learning and quick adaptation for embedding imputations is a desired property. One can combine KG2Vec with meta-learning, e.g., MAML in Finn et al. (2017), such that the resulting model can quickly learn the embeddings of newly added nodes (words), or updated node features.

\section{Conclusion and Future Work}

In this paper, we introduce $\mathrm{KG} 2 \mathrm{Vec}$, a graph neural network based approach for embedding imputation of OOV words which makes use of grounded language information. Using publicly available information sources like Wikipedia and Wiktionary, KG2Vec can effectively impute embeddings for rare or unseen words. Experimental results show that KG2Vec achieves state-ofthe-art results on the Card-660 dataset. Future research directions include a theoretical explanation of KG2 Vec and applications to downstream NLP tasks.

\section{Acknowledgments}

We would like to thank the anonymous reviewers for their valuable feedback.

\section{References}

Dzmitry Bahdanau, Tom Bosc, Stanislaw Jastrzebski, Edward Grefenstette, Pascal Vincent, and Yoshua Bengio. 2017. Learning to compute word embeddings on the fly. CoRR, abs/1706.00286.

Antoine Bordes, Sumit Chopra, and Jason Weston. 2014. Question answering with subgraph embeddings. CoRR, abs/1406.3676.

Jos de Bruijn, Marc Ehrig, Cristina Feier, Francisco MartnsRecuerda, francois Scharffe, and Moritz Weiten. 2006. Ontology Mediation, Merging, and Aligning, pages 95 - 113 .
Eunsol Choi, He He, Mohit Iyyer, Mark Yatskar, Wentau Yih, Yejin Choi, Percy Liang, and Luke Zettlemoyer. 2018. Quac: Question answering in context. CoRR, abs/1808.07036.

Chelsea Finn, Pieter Abbeel, and Sergey Levine. 2017. Model-agnostic meta-learning for fast adaptation of deep networks. In Proceedings of the 34th International Conference on Machine Learning-Volume 70, pages 1126-1135. JMLR. org.

Thomas R. Gruber. 1993. A translation approach to portable ontology specifications. Knowl. Acquis., 5(2):199-220.

N. Guarino. 1998. Formal Ontology in Information Systems: Proceedings of the 1st International Conference June 6-8, 1998, Trento, Italy, 1st edition. IOS Press, Amsterdam, The Netherlands, The Netherlands.

Sanda M. Harabagiu, Dan I. Moldovan, Christine Clark, Mitchell Bowden, Andrew Hickl, and Patrick Wang. 2005. Employing two question answering systems in trec 2005. In TREC.

He He, Anusha Balakrishnan, Mihail Eric, and Percy Liang. 2017. Learning symmetric collaborative dialogue agents with dynamic knowledge graph embeddings. CoRR, abs/1704.07130.

Aurélie Herbelot and Marco Baroni. 2017. High-risk learning: acquiring new word vectors from tiny data. CoRR, abs/1707.06556.

Thomas N Kipf and Max Welling. 2016. Semisupervised classification with graph convolutional networks. arXiv preprint arXiv:1609.02907.

Thang Luong, Richard Socher, and Christopher Manning. 2013. Better word representations with recursive neural networks for morphology. In Proceedings of the Seventeenth Conference on Computational Natural Language Learning, pages 104-113. Association for Computational Linguistics.

Tomas Mikolov, Ilya Sutskever, Kai Chen, Greg Corrado, and Jeffrey Dean. 2013. Distributed representations of words and phrases and their compositionality. In Proceedings of the 26th International Conference on Neural Information Processing Systems Volume 2, NIPS'13, pages 3111-3119, USA. Curran Associates Inc.

Jane Morris and Graeme Hirst. 1991. Lexical cohesion computed by thesaural relations as an indicator of the structure of text. Comput. Linguist., 17(1):2148.

Ian Niles and Adam Pease. 2003. Linking lexicons and ontologies: Mapping wordnet to the suggested upper merged ontology. In Proceedings of the 2003 International Conference on Information and Knowledge Engineering (IKE 03), Las Vegas, pages 412-416. 
Ekaterina Ovchinnikova, Laure Vieu, Alessandro Oltramari, Stefano Borgo, and Theodore Alexandrov. 2010. Data-driven and ontological analysis of framenet for natural language reasoning. In Proceedings of the Seventh International Conference on Language Resources and Evaluation (LREC'10), Valletta, Malta. European Language Resources Association (ELRA).

Heiko Paulheim. 2017. Knowledge graph refinement: A survey of approaches and evaluation methods. Semantic Web, 8(3):489-508.

Jeffrey Pennington, Richard Socher, and Christopher D. Manning. 2014. Glove: Global vectors for word representation. In In EMNLP.

Mohammad Taher Pilehvar and Nigel Collier. 2017. Inducing embeddings for rare and unseen words by leveraging lexical resources. In Proceedings of the 15th Conference of the European Chapter of the Association for Computational Linguistics: Volume 2, Short Papers, pages 388-393. Association for Computational Linguistics.

Mohammad Taher Pilehvar, Dimitri Kartsaklis, Victor Prokhorov, and Nigel Collier. 2018. Card-660: Cambridge Rare Word Dataset - a reliable benchmark for infrequent word representation models. In Proceedings of the 2018 Conference on Empirical Methods in Natural Language Processing, Brussels, Belgium.

Yuval Pinter, Robert Guthrie, and Jacob Eisenstein. 2017. Mimicking word embeddings using subword rnns. CoRR, abs/1707.06961.

Michael Schlichtkrull, Thomas N Kipf, Peter Bloem, Rianne Van Den Berg, Ivan Titov, and Max Welling. 2018. Modeling relational data with graph convolutional networks. In European Semantic Web Conference, pages 593-607. Springer.

Rico Sennrich, Barry Haddow, and Alexandra Birch. 2015. Neural machine translation of rare words with subword units. CoRR, abs/1508.07909.

Robert Speer, Joshua Chin, and Catherine Havasi. 2017. Conceptnet 5.5: An open multilingual graph of general knowledge. In Thirty-First AAAI Conference on Artificial Intelligence.

Keyulu Xu, Weihua Hu, Jure Leskovec, and Stefanie Jegelka. 2018. How powerful are graph neural networks? arXiv preprint arXiv:1810.00826.

Kun $\mathrm{Xu}$, Yansong Feng, Songfang Huang, and Dongyan Zhao. 2016. Hybrid question answering over knowledge base and free text. In COLING.

Zhitao Ying, Jiaxuan You, Christopher Morris, Xiang Ren, Will Hamilton, and Jure Leskovec. 2018. Hierarchical graph representation learning with differentiable pooling. In Advances in Neural Information Processing Systems, pages 4805-4815.
Jinman Zhao, Sidharth Mudgal, and Yingyu Liang. 2018. Generalizing word embeddings using bag of subwords. CoRR, abs/1809.04259. 